\title{
Real-time PCR Analysis of RNA Extracted From Formalin-fixed and Paraffin-embeded Tissues: Effects of the Fixation on Outcome Reliability
}

\author{
Francesca Castiglione, MD,* Duccio Rossi Degl'Innocenti, BS, ${ }^{*}$ Antonio Taddei, MD, $\dagger$ \\ Francesca Garbini, MD,* Anna Maria Buccoliero, MD,* Maria Rosaria Raspollini, MD,* \\ Monica Pepi, BS, * Milena Paglierani, BS,* Grazia Asirelli, BS, ${ }^{*}$ Giancarlo Freschi, MD, $\dagger$ \\ Paolo Bechi, MD, $\dagger$ and Gian Luigi Taddei, MD*
}

\begin{abstract}
In many pathologic circumstances, quantitative mRNA expression levels are important for evaluation of possible genome mutations. The development of real-time polymerase chain reaction (RT-PCR) technology has facilitated the realization of nucleic acid quantification. Potentially, quantitative PCR offers a number of advantages over traditional methods because it permits the use of small amounts of genetic material. In the present study, we optimize a RNA purification technique on specimens that are formalin-fixed, paraffin-embedded and we examine prolonged formalin fixation effects on quantitative RT-PCR analysis. We compared RNA levels with 70 colic mucosa samples using the cyclooxygenase 2 gene as marker. The difference in amplification successes between formalin-fixed tissues and formalin-fixed, paraffin-embedded tissues was not statistically significant. Moreover, we compared the expression of formalin-fixed samples with the expression of each fresh tissue. Wilcoxon Mann-Whitney test shows that only the difference in the expression levels of 1- or 3hour formalin-fixed samples is not statistically significant with respect to other fixation times. We found that the mRNA can be reliably extracted from formalin fixed, paraffin-embedded tissue sections but that prolonged formalin fixation produces different results in quantitative RT-PCR. It can be related to difference in RNA sequences length and the generation of secondary structures that are more susceptible to the prolonged formalin fixation. We suppose that the paraffin do not influence the RNA extraction yield because there are no statistical significant differences between amplification success of formalin-fixed tissues and paraffin-embedded tissues. Therefore, in relative expression quantization, we confirm that it is appropriate to use specimens with same protocols and time for formalin fixation.
\end{abstract}

Key Words: real-time PCR, RNA, extracted, formalin-fixed

(Appl Immunohistochem Mol Morphol 2007;15:338-342)

Received for publication January 4, 2006; accepted April 14, 2006.

From the *Department of Human Pathology and Oncology; and $\dagger$ Surgical Pathology 1, University of Florence, School of Medicine, Florence, Italy.

Reprints: Duccio Rossi Degl'Innocenti, BS, Department of Human Pathology and Oncology, University of Florence, School of Medicine, Viale GB Morgagni, 85. 50134 Florence, Italy (e-mail: duccio.rossideglinnocenti@unifi.it).

Copyright (C) 2007 by Lippincott Williams \& Wilkins
$T$ he archives of departments of pathology represent an unique source of morphologically defined tissues derived from normal and pathologically altered tissues for which extensive clinical data are available. Most pathologic specimens are routinely formalin-fixed and paraffin-embedded before histologic evaluation.

Although tissue architecture and proteins are preserved, extraction of nucleic acids may be difficult, yielding degraded DNA only. However, these samples form an invaluable resource for molecular studies with clinical correlation.

The feasibility of performing molecular genetic analysis of fixed and paraffin-embedded tissues will improve diagnostic procedures and will enable a large number of research studies on various diseases. ${ }^{1}$

The most widely used fixative in pathology is formalin. It is comparably cheap, easy to handle, provides superior morphologic quality, and is compatible with nearly all relevant antibodies for immunohistochemical staining. The condition of fixation, as time and temperature, changes for many tissues and for many operators.

The fixation of tissue sample in formaldehyde leads to extensive crosslinking of all tissues components. As a consequence of this crosslinking, the nucleic acids isolated from these specimens are highly fragmented. The extent of fragmentation depends on the tissue type and the condition of fixation. ${ }^{2,3}$ On the other hand, the process of formalin fixation causes cross-linkage of nucleic acids with proteins and covalently modifies RNA.,3

In many pathologic circumstances, quantitative mRNA expression levels are important for evaluation of possible genome mutations.

The development of real-time polymerase chain reaction (RT-PCR) technology has greatly facilitated the realization of nucleic acid quantification. Potentially, quantitative PCR offers a number of advantages over traditional methods because it permits the use of relatively small amounts of genetic material that may be fragmented or degraded, such as that obtained from routinely processed tissue sections.

The theory and practice of this new methodology have been extensively discussed in several recent reviews. $^{5-9}$ 
However, these methods are limited by finding unfixed tissues to permit isolation of high-quality RNA. ${ }^{12}$ Recently, many laboratories developed different methods for RNA extraction from paraffin-embedded tissue with various results. ${ }^{10-15}$

Recent works asserts that RNA extracted from formalin-fixed and paraffin-embedded tissue can be used for quantitative analysis ${ }^{16-21}$ whereas Foss et al $^{2}$ indicate a limited utility for retrospective studies of archival tissues.

In the present study, we optimize a deparaffinization and RNA purification techniques, with appreciable results.

The purpose of this study was to examine of prolonged formalin fixation effects on quantitative RTPCR analysis. Then we evaluated fragmentation state, RNA extraction yield and results reliability according to different time of formalin fixation of surgical specimens. In consequence, we analyzed mRNA in RT-PCR using glyceraldehyde-3-phosphate dehydrogenase (GAPDH) as housekeeping gene and cyclooxygenase-2 gene (COX-2).

\section{MATERIALS AND METHODS}

\section{Tissues}

Full thickness colon parietal fragments from 10 consecutive patients ( 6 men, 4 women, mean age 68 years) were obtained by cold blade cut from the operative specimens of patients undergoing colonic resection for cancer. Specimens were taken at least $10 \mathrm{~cm}$ far away from the neoplasia, and colonic mucosa seemed normal at macroscopic evaluation. Patients did not receive preoperative radiotherapy or chemotherapy and were treated at the Surgical Pathology 1, University of Firenze.

All samples were microscopically evaluated and classified as normal colonic mucosa.

\section{Tissue Fixation}

From each of the 10 fresh normal colon mucosa specimens, we cut 7 small fragments macroscopically representative of the tissue. All tissue pieces were sectioned to ensure that all spatial dimensions were $\leq 10 \mathrm{~mm}(10 \times 10 \times 0.3 \mathrm{~mm})$. All diagnoses were confirmed by examination of $5 \mu \mathrm{m}$ hematoxylin and eosinstained sections to ensure that all tissues were similarly representative. To have the minimum risk of COX-2 gene expression differences, we have carefully analyzed and chosen fragments of samples which were as similar as possible for histomorphologic structure and for amount and pattern of blood vessels. To minimize contamination, a new surgical blade was used for each specimen. A fragment was immersed in RNAlater (Qiagen, Hilden, Germany) so kept overnight at $4^{\circ} \mathrm{C}$ and stored at $-80^{\circ} \mathrm{C}$ until analyzed. Three fragments were fixed in buffered formalin 10\% (Bio-Optica, Milan, Italy) for 1, 3, and 24 hours, respectively, and then were immersed in RNAlater (Qiagen, Hilden, Germany) as the previous sample. The other 3 fragments were fixed in buffered $10 \%$ formalin for 24,48 , and 72 hours, respectively, and paraffin-embedded routinely with Tissue Processor Citadel 2000 (ThermoShandon, Runcorn, UK).

\section{RNA Isolation}

Sections $(10 \mu \mathrm{m})$ were microtome cut; the microtome was cleaned after each use. Three colon tissue sections from formalin-fixed, paraffin-embedded samples were subjected to xylene/ethanol deparaffinization. In brief, $1 \mathrm{~mL}$ of xylene for sample was added and centrifuged (5 minutes at $10 \mathrm{rpm}$ ); the supernatant was removed and the step was repeated. Then, we carried out 3 identical washes with ethanol $100 \%, 70 \%$, and $50 \%$. The tissue pellet was air-dried. The samples were resuspended in $200 \mu \mathrm{L}$ RNA lysis Buffer RTL (RNasy Fibrous Tissue Mini Kit-Qiagen, Hilden, Germany) and homogenized using PCR Tissue Homogenizing kit (PBI International, Milan, Italy) in microcentrifuge tubes. RNA was isolated using RNasy Fibrous Tissue Mini Kit (Qiagen, Hilden, Germany) and successively stored at $-80^{\circ} \mathrm{C}$

The samples not treated with paraffin embedding were defrosted and cut into small pieces: homogenization and RNA isolation were developed by the previous method.

RNAs were quantified by spectrophotometer at OD of $260 \mathrm{~nm}$, and A260/A280 ratios > 1.8 were considered to be of high purity. ${ }^{2}$ RNA fragmentation state was evaluated by $1 \%$ agarose gel.

\section{Real-Time quantitative PCR}

All RNA samples (500 ng) were reverse transcribed to cDNA using High-capacity cDNA Archive Kit (Applied Biosystems, Foster City) according to the manufacturer's protocol with little modifications: the reaction was carried out with $5 \mu \mathrm{L}$ of $10 \mathrm{X}$ RT Buffer, $2 \mu \mathrm{L}$ of $25 \mathrm{X}$ dNTPs, $5 \mu \mathrm{L}$ of $10 \mathrm{X}$ random primers, $2.5 \mu \mathrm{L}$ of MultiScribe Reverse Trascriptase $(50 \mathrm{U} / \mu \mathrm{L})$, and $10.5 \mu \mathrm{L}$ of nuclease-free water.

In the RT reaction negative controls were performed. TaqMan real-time quantitative PCR was performed on an ABI PRISM 7000 Sequence Detector System (Applied Biosystems, Foster City). Procedure used to detect specific RNA molecules frequently require the use of internal positive controls, typically on the basis of housekeeping gene mRNAs. Housekeeping genes are constitutively expressed in all tissues, usually serve basic metabolic functions in the cell, and are highly conserved throughout evolution. The ideal housekeeping gene control message is one whose expression in a particular tissue is constant across a wide range of physiologic condition. To evaluate further the potential of this technique, we compared an established housekeeping gene and a target gene mRNA controls, GAPDH and COX-2 expression, in evaluating the effects of varying fixation times on the recovery and subsequent RT-PCR amplification of RNA from paraffin-embedded tissues .

PCR products for COX-2 were detected using genespecific primers and probes labeled with reporter day FAM (Assay on Demand, Applied Biosystems, Foster 
City). GAPDH was used as endogenous control gene for normalization and was detected using gene-specific primers and probes labeled with reporter day VIC (Applied Biosystems, Foster City).

Amplification products length of these assays are less than 100 base pairs, therefore they work likely with fragmented RNA too.

PCR reaction was carried out in triplicate on 96well plate with $20 \mu \mathrm{L}$ per well using 1x TaqMan Universal PCR MasterMix. After an incubation for 2 minutes at $50^{\circ} \mathrm{C}$ and 10 minutes at $95^{\circ} \mathrm{C}$, the reaction continue for 50 cycles at $95^{\circ} \mathrm{C}$ for 15 seconds and $60^{\circ} \mathrm{C}$ for 1 minute.

\section{Results Analysis}

At the end of the reaction, the results were evaluated using the ABI 7000 PRISM software and the $\mathrm{Ct}$ values were exported to Microsoft Excel. The $\mathrm{Ct}$ values for each set of 3 reactions were averaged for all subsequent calculations.

The $2^{-\Delta \Delta \mathrm{Ct}}$ method described by Livak and Schmittgen et $\mathrm{al}^{22}$ was used to analyze the results. As control samples, for the 6 samples derived from each original specimen, we used the tissues not fixed in formalin. With this method, we have value equal to 1 when no change in relative expression occurred with respect to tissue unfixed. We defined over-expression when $2^{-\Delta \Delta \mathrm{Ct}}>1$ and under-expression when $2^{-\Delta \Delta \mathrm{Ct}}<1$.

\section{Statistical Analysis}

The shift of relative expression level of $\mathrm{COX}-2$, in colic mucosa was calculated according to the Wilcoxon Mann-Whitney test. Data analysis was performed using SPSS Version 11.0 (Chicago, IL) statistical package. Fisher exact test was applied to statistical analysis of amplification's return. A $P$ value $\leq 0.05$ was considered to be statistically significant.

\section{RESULTS}

\section{RNA Extraction}

We extracted RNA from 64 of 70 samples. The RNA concentration and purity in this preparation was quantitated spectrophotometrically by measuring their absorbance at 260 and $280 \mathrm{~nm}$. RNA from fresh and formalin-fixed tissues was isolated in all cases. The RNA fragmentation state was estimated with ethidium bromide-stained $1 \%$ agarose gel (Fig. 1). The fragment length of RNA was around 130 to $200 \mathrm{bp}$ for the formalin-fixed samples and $>650 \mathrm{bp}$ for the fresh tissues.

\section{RT-PCR}

We compared RNA levels to 70 colic mucosa samples using the marker COX-2. The RNA isolated were transcribed to cDNA in all cases and amplified by RT-PCR in 61 cases (Table 1). The difference in amplification successes between formalin-fixed tissues and formalin-fixed, paraffin-embedded tissues was not statistically significant (Fisher exact test $P=0.472$ ). RNA signal was not detected for any marker in negative controls. We compared the expression of formalin-fixed

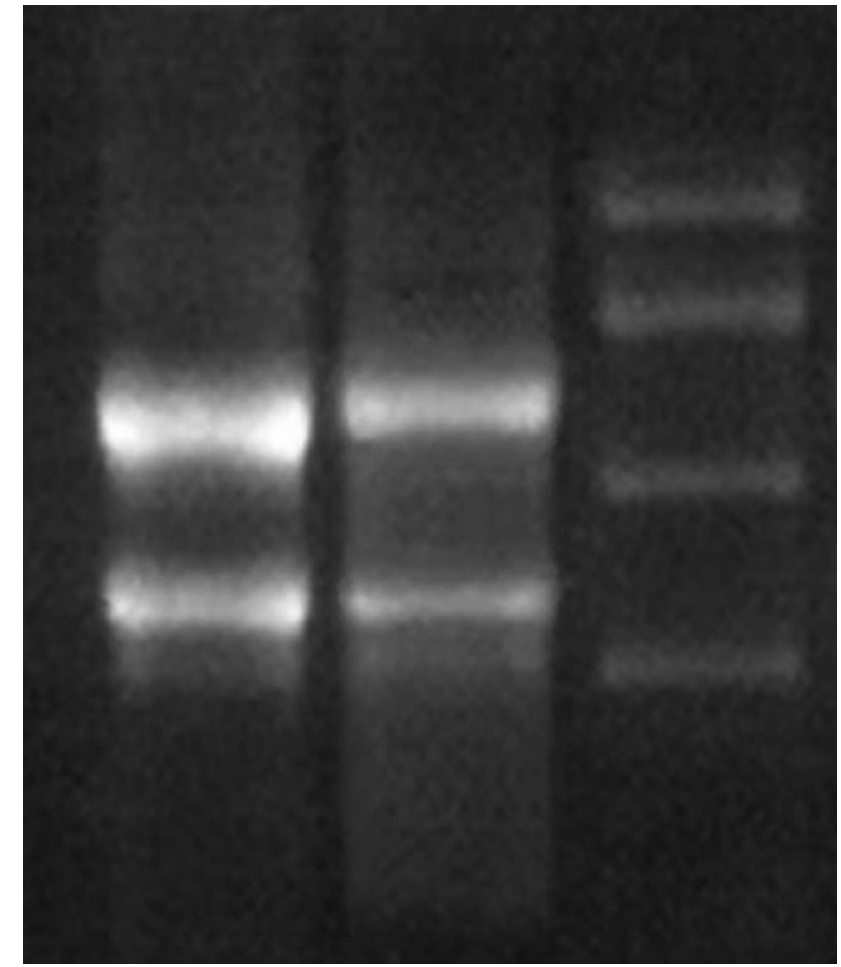

FIGURE 1. RNA fragmentation state estimated with ethidium bromide-stained 1\% agarose gel: in left line is shown RNA from fresh tissue; in right is line shown RNA from FFPE tissue.

samples with the expression of each fresh tissue. Results are shown in Table 1, where a value $>1$ represent a overexpression and a value between 0 and 1 represent a underexpression respect to fresh tissue (value $=1$ ). COX-2 was over-expressed in 3/10 (30\%) 1-hour formalin-fixed colic mucosa tissues, for 3-hour formalin-fixed, COX-2 was over-expressed in 3/9 (33\%) and for 24-hour formalinfixed was over-expressed in $7 / 8(87 \%)$ tissues. For 24hour formalin-fixed paraffin-embedded tissues, COX-2 was over-expressed in $7 / 9(77 \%)$, for 48 hours it was $6 / 8$ $(75 \%)$ and for 72 hours $6 / 7(86 \%)$.

The average of the values show over-expression in all the cases with the exception of 3-hour formalin-fixed tissues which show under-expression. An important overexpression shown in $\geq 24$-hour formalin-fixed, paraffinembedded tissue (Fig. 2).

Wilcoxon Mann-Whitney test shows that the difference the expression levels of 1 or 3-hour formalinfixed samples is not statistically significant $(P>0.05)$, whereas the difference of other samples is significant $(0.003>P<0.05)$.

\section{DISCUSSION}

Large numbers of formalin-fixed and paraffinembedded tissue samples have been collected throughout the years and stored in pathology departments to verify the preliminary diagnosis of various diseases. Given the 
TABLE. 1 COX-2 Relative Expression Levels of Formalin-fixed Tissues Compared with Value of Fresh Tissues

\begin{tabular}{|c|c|c|c|c|c|c|c|}
\hline CASE & Fresh & $1 \mathrm{~h} \mathrm{FF}$ & 3 h FF & $24 \mathrm{~h} \mathrm{FF}$ & $24 \mathrm{~h}$ FF/PE & $48 \mathrm{~h}$ FF/PE & 72 h FF/PE \\
\hline $\mathrm{C} 1$ & 1.00 & 0.64 & 0.55 & 1.22 & 1.42 & 1.98 & 5.20 \\
\hline $\mathrm{C} 2$ & 1.00 & 0.56 & 0.82 & 0.99 & 1.01 & 2.19 & 12.74 \\
\hline C3 & 1.00 & 1.07 & 0.34 & - & 0.56 & - & 1.22 \\
\hline $\mathrm{C} 4$ & 1.00 & 0.98 & 1.10 & 7.02 & 1.25 & 7.16 & 4.70 \\
\hline $\mathrm{C} 5$ & 1.00 & 1.24 & 1.56 & 3.01 & 2.91 & - & 5.81 \\
\hline C6 & 1.00 & 0.76 & 0.56 & 2.40 & 5.83 & 1.14 & - \\
\hline C7 & 1.00 & 2.53 & 0.33 & - & 0.90 & 0.74 & - \\
\hline $\mathrm{C} 8$ & 1.00 & 2.10 & 0.89 & 12.11 & - & 2.03 & 10.08 \\
\hline C9 & 1.00 & 0.76 & 2.05 & 6.55 & 5.50 & 0.78 & 0.97 \\
\hline $\mathrm{C} 10$ & 1.00 & 0.58 & - & 1.12 & 9.24 & 6.79 & - \\
\hline
\end{tabular}

The Dash Means an Amplification Failure.

FF indicates formalin-fixed; PE indicates paraffin-embedded.

wide availability of these archival tissues, the whole spectra of diseases can be studied retrospectively. Therefore, the analysis of archival tissue becomes valuable for molecular biology investigations. Today one of the most investigated topics is gene expression. Studying of gene expression can be performed using different approaches such as RT-PCR.

Quantitative RT-PCR can determine gene alterations. It can be used for monitoring minimal residual disease and chimerism in patients after allogenic transplantation, and to quantify cytochine gene expression. It can also be used for detection of measles virus RNA in paraffin-embedded tissue and for analysis of T-cell receptor $\beta$-chain. ${ }^{23-28}$

The purpose of this study was to examine the effect of prolonged formalin fixation on quantitative RT-PCR analysis. We combined the extraction method of formalin-fixed, paraffin embedded tissues with the RT-PCR. This technique demands a very little amount but a goodquality RNA.

We found that the mRNA can be reliably extracted from formalin-fixed, paraffin-embedded tissue sections

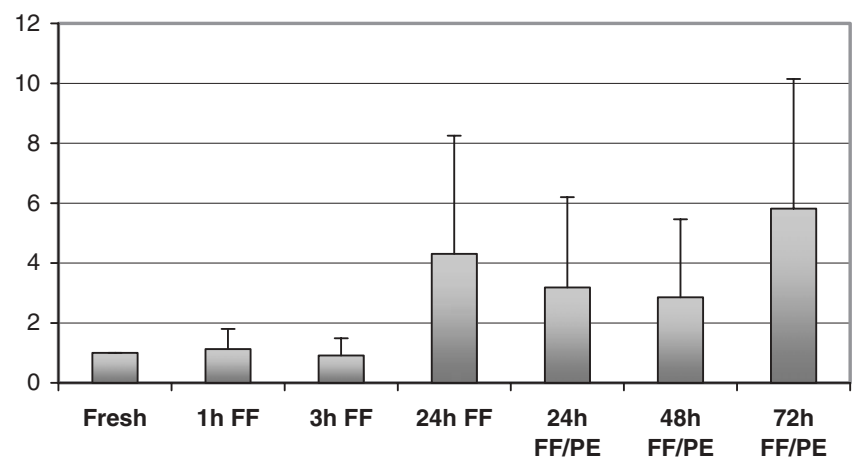

FIGURE 2. Effects of fixation times on relative expression levels for COX-2 in colic mucosa. The bars indicate the mean expression level for each different fixation time. All values were compared with fresh tissue value. The difference in expression levels of 1- or 3-hour formalin-fixed samples is not statistically significant $(P>0.05)$, whereas the difference of other samples is significant (Wilcoxon Mann-Whitney test $0.003>P<0.05$ ). but that prolonged formalin fixation products different results in quantitative RT-PCR.

As many works showed previously ${ }^{29-31}$ COX-2 expression was present in colic mucosa samples.

In 24-hour formalin-fixed samples and $\geq 24$-hour formalin-fixed paraffin-embedded samples, COX-2 expression levels show marked and statistically significant alterations. These differences in the results obtained using the same material, is difficult to understand. It can be related to difference in RNA sequences length and the generation of secondary structures that are more susceptible to the effects of prolonged formalin fixation. Otherwise it can be related with the differences in amplicon length of 2 genes analyzed (GAPDH and COX-2) or with the different formalin effects on secondary RNA structures of these genes.

Moreover, there are no statistically significant differences between amplification success of formalinfixed only and paraffin-embedded tissues. So we can suppose that the paraffin does not influence the RNA extraction yield.

Furthermore, COX-2 expression levels in 24-hour formalin-fixed tissues and in 24-hour formalin-fixed paraffin-embedded tissues, are not significant $(P=0.41)$. This shows that the paraffin does not influence gene expression analysis.

Our results showed significant differences in relative expression in the mean of samples fixed with different duration, 1 and 24 hours. However, our aim was to find good conditions of fixation duration to preserve tissue samples, and make them available for both morphologic and bio-molecular analyses. Formalin fixation should be considered the shortest time needed to better preservation of tissue morphology for a correct anatomo-pathologic evaluation. Therefore, we suggest that optimal fixation time, needed for a good preservation of both tissue morphology and nucleic acids for bio-molecular analysis, is 24 hours which is not always possible; various factors influence the fixation time in a department of pathology, like size of specimens and protocols of immunohistochemical analysis: it increases the fixation times indefinitely. We suppose the RNA is more fragmentary after 
24-hour formalin fixation. But it is still suitable for molecular analysis provided that the fixation time does not overtakes 72 hours. After this time, the results may not be reliable. If samples have been fixed for more than 24 hours they can be analyzed and compared for a relative quantization. It can be done only if all samples were fixed using the same. Otherwise, if we perform a relative expression quantization using different times formalin-fixed samples, the results may be not reliable. For this reason, it is necessary to standardize the fixation protocols of specimens in a department of pathology.

Our results show that the RNA extraction, retrotranscription to cDNA and quantitative RT-PCR investigations should be feasible in many cases with the protocol we described here. Actually these results confirm the suitability of quantitative RT-PCR for determining relative mRNA expression in formalin-fixed paraffinembedded tissues.

\section{REFERENCES}

1. Mizuno T, Nagamura H, Iwamoto KS, et al. RNA from decadesold archival tissue blocks for retrospective studies. Diagn Mol Pathol. 1998;7:202-208.

2. Foss RD, Guha-Thakurta N, Conran RM, et al. Effects of fixative and fixation time on the extraction and polymerase chain reaction amplification of RNA from paraffin-embedded tissue. Comparison of two housekeeping gene mRNA controls. Diagn Mol Pathol. 1994;3:148-155.

3. Paska C, Bogi K, Szilak L, et al. Effect of formalin, acetone, and RNAlater fixatives on tissue preservation and different size amplicons by real-time PCR from paraffin-embedded tissue. Diagn Mol Pathol. 2004;13:234-240.

4. Masuda N, Ohnishi T, Kawamoto S, et al. Analysis of chemical modification of RNA from formalin-fixed sample and optimization of molecular biology applications for such samples. Nucleic Acids Res. 1999;27:4436-4443.

5. Bernard PS, Wittwer CT. Real-time PCR technology for cancer diagnostics. Clin Chem. 2002;48:1178-1185.

6. Freeman WM, Walker SJ, Vrana KE. Quantitative RT-PCR Pitfalls and potential. Biotechniques. 2002;26:112-125.

7. Lewis F, Maughan NJ, Smith V, et al. Unlocking, the archive-gene expression in paraffin-embedded tissue. $J$ Pathol. 2001;195:66-71.

8. Mocellin S, Rossi CR, Marincola FM. Quantitative real-time PCR in cancer research. Arch Immunol Ther Exp. 2003;51:301-313.

9. Ong YL, Irvine A. Quantitative real-time PCR: a critique of method and practical considerations. Hematology. 2002;7:59-67.

10. Coombs NJ, Gough AC, Primrose JN. Opimisation of DNA and RNA extraction from archival formalin-fixed tissue. Nucl Acid Res. 1999;27:e12.

11. Koopmans M, Monroe SS, Coffield LM, et al. Optimization of extraction and PCR amplification of RNA extracts from paraffinembedded tissue in different fixatives. J Virol Meth. 1993;43: 189-204.

12. Körbler T, Gršković M, Dominis $\mathrm{M}$, et al. A simple method for RNA isolation from formali-fixed and paraffin-embedded lymphatic tissues. Exp Mol Pathol. 2003;74:336-340.
13. Lehmann U, Kreipe H. Real-Time PCR analysis of DNA and RNA extracted from formalin-fixed and paraffin-embedded biopsies. Methods. 2001;25:409-418.

14. Rupp GM, Locker J. Purification and analysis of RNA from paraffin-embedded tissues. Biotechniques. 1988;29B:3-7.

15. Stanta G, Schneider C. RNA extracted from paraffin-embedded human tissues is amenable to analysis by PCR amplification. Biotechniques. 1991;11:304-308.

16. Cairns MT, Church S, Jhonston PG, et al. Paraffin-embedded tissue as a source of RNA for gene expression analysis in oral malignancy. Oral Dis. 1997;3:501-509.

17. Finke J, Fritzen R, Ternes $P$, et al. An improved strategy and a useful housekeeping gene for RNA analysis from formalinfixed, paraffin-embedded tissues by PCR. Biotechniques. 1993; 14:448-453.

18. Gloghini A, Canal B, Klein U, et al. RT-PCR analysis of RNA extracted from Bouin-fixed and paraffin-embedded lymphoid tissues. J Mol Diagn. 2004;6:290-296.

19. Godfrey TE, Kim SH, Chavira M, et al. Quantitative mRNA expression analysis from formali-fixed, paraffin-embedded tissues using 5' nuclease quantitative reverse transcription-polymerase chain reaction. $J$ Mol Diagn. 2000;2:84-91

20. Macabeo-Ong M, Ginzinger DG, Dekker N, et al. Effect of duration of fixation on quantitative reverse transcription polymerase chain reaction analyses. Mod Pathol. 2001;15:979-987.

21. Specht K, Richter T, Muller U, et al. Quantitative gene expression analysis in microdissected archival formali-fixed and paraffinembedded tumor tissue. Am J Pathol. 2001;158:419-429.

22. Livak KJ, Schmittgen TD. Analysis of relative gene expression data using real-time quantitative PCR and the $2(-\Delta \Delta \mathrm{C}(\mathrm{T}))$ method. Methods. 2001;25:402-408.

23. Elmaagacli AH. Real-time PCR for monitoring minimal residual disease and chimerism in patients after allogeneic transplantation. Int J Hematol. 2002;76:204-205.

24. Giulietti A, Overbergh L, Valckx D, et al. An overview of real-time quantitative PCR: applications to quantify cytokine gene expression. Methods. 2001;25:386-401.

25. Jackson DP, Quirke P, Lewis F, et al. Detection of measles virus RNA in paraffin-embedded tissue. Lancet. 1989;1:1391-1399.

26. O'Shea U, Wyatt JI, Howdle PD. Analysis of T cell receptor $\beta$-chain CDR3 size using RNA extracted from formalin fixed paraffin wax embedded tissue. J Clin Pathol. 1997;50:811-814.

27. Svoboda-Newman SM, Greenson JK, Singleton TP, et al. Detection of hepatitis C by RT-PCR in formalin-fixed paraffin-embedded tissue from liver transplant patients. Diagn Mol Pathol. 1997;6: $123-129$

28. Van der Velden VH, Hochhaus A, Cazzaniga G, et al. Detection of minimal residual disease in hematologic malignancies by real-time quantitative PCR: principles, approaches, and laboratory aspects. Leukemia. 2003;17:1013-1034.

29. Einspahr JG, Krouse RS, Yokim JM, et al. Association between cyclooxygenase expression colorectal adenoma characteristics. Cancer Res. 2003;63:3891-3893.

30. Reddy BS, Hirose J, Lubet R, et al. Chemoprevention of colon cancer by specific cyclooxygenase-2 inhibitor, celecoxib, administered during different stages of carcinogenesis. Cancer Res. 2000;60:293-297.

31. Sheehan KM, O'Connell F, O'Grady A, et al. The relationship between cyclooxygenase -2 expression and characteristics of malignant transformation in human colorectal adenomas. Eur $J$ Gastroenterol Epathol. 2004;16:619-625. 